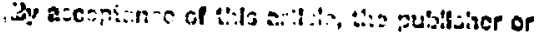

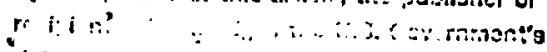

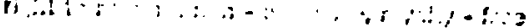

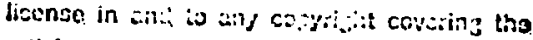

cricle.

\title{
IMPURITY AND SURFACE STUDIES IN ORMAK*
}

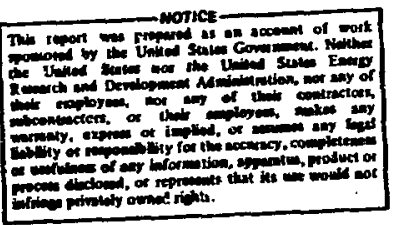

R. J. Colchin, C. E. Bush, G. L. Jahns, J. F. Lyon,

M. Murakami, R. V. Neidigh, and D. L. Shaeffer

Oak Ridge National Laboratory

Oak Ridge, Tennessee 37830 , USA

\section{Abstract}

The ORMAK tokamak has been in operation since 1971, and surface impurity problems have been pursued from the beginning. Surface studies of materials removed from ORMAK have revealed the presence of $\mathrm{C}, 0$, and Fe. These are also the principal impurities observed spectroscopically in plasma discharges, although numerous other elements are present in lesser amounts. Spectroscopy, x-ray measurements, plasma resistance, and fast ion scattering have been used in an effort to determine $z_{\text {eff }}$, the effective nuclear charge of plasma ions. All four measurements have practical difficulties leading to relatively large experimental error 1imits. Oxygen discharge pre-cleaning has allowed ORMAK discharges to reach higher currents and correspondingly higher ion and electron temperatures; spectroscopic studies reveal a lower level of contaminants, particularly $C$ and $N$. Power measurements indicate that most of the input power strikes the walls, mostly as radiation. By varying operating parameters it is found that $z_{\text {eff }} \sim I_{p} / \bar{n}_{e}$.

\footnotetext{
* Research sponsored by the Energy Research and Development Administration under contract with the Union Carbide Corporation.
} 


\section{Introduction}

Surface and impurity problems have been actively pursued using the ORMAK tokamak since its inception. As a precaution against the adsorption of gases, the stainless steel walls of the liner were vacuum coated with a layer of platinum and gold $[1,2]$, with the $\mathrm{Pt}$ acting as a seal to prevent impurities in the stainless steel from reaching the outer Au coating. In spite of such clean beginnings, the liner walls are presently contaminated with elements other than gold. Our understanding of this contamination is detailed in the next section. Details of the resulting plasma impurities and impurity measurements are given in Section 3 . The consequences of oxygen discharge pre-cleaning are shown in Section 4, and Section 5 discusses measurements of power to the walls and impurity scaling.

\section{Surface Contamination}

Surface contamination on the walls of the ORMAK liner has been identified by visual inspection, by Auger electron spectroscopy (AES) of removed wall samples, and by in situ measurements using a soft $x$-ray appearance potential spectrometer (SXAPS). The SXAPS experiment is described in another paper [3].

Visible contamination arising from two sources has been identified. Near the limiter, gold on the walls is covered with tungsten, encompassing approximately a third of the surface area of the torus. Gold remains visible in areas shielded by small liner protrusions, indicating that tungsten has been deposited at grazing incidence to the walls. In the region of the Thomson scattering system, $180^{\circ}$ away from the limiter, several dark areas are evident. Again, these dark areas are in slight depressions, between seams in the liner. They are centered about the 
region of the laser viewing dump, which consists of tightly packed austenitic stainless steel razor blades. Liner samples taken from nearby show $\varangle 50 \%$ coverage with $\mathrm{Fe}$, so that it is likely that the dark arcas are deposits of iron.

Various plugs, lenses, and other parts of the first 1 iner and the present (second) liner have been analyzed by AES. All such analyses involved exposure to air after removal from ORMAK, with probable accompanying surface contaminatios. Ion sputtering has been used to remove surface layers and thereby provide depth profiles. The principal contaminants found were $\mathrm{C}, \mathrm{O}$, and $\mathrm{Fe}[4]$.

\section{ORMAK Plasma Contamination and $Z$ eff}

The first indications of plasma impurities came from optical observations. The extent to which impurities cause energy loss and control plasma current profiles was rather dimly appreciated until three to four years ago. Since that time a lot of effort has been expended to identify and determine the amount of impurities in the plasma [5], the effect of impurities on both particle transport and energy loss, and the manner in which walls react with the plasma. Unfortunately, there is as yet no easy way to measure any of these phenomena, so that only partial answers are possible.

In fact, the determination of $Z_{\text {eff }}$, $z_{\text {eff }}=\sum_{i} n_{i} z_{i}^{2} / n_{e}$,

(i indicates ion species, $n_{i}$ and $n_{e}$ are ion and electron densities, $z_{i}$ is the charge state of the ion), by any of several means is not a simple task; and it is often difficult to determine whether a change in wall contamination (such as by oxygen discharge cleaning, discussed in Sect. 4) 
increases or decreases $Z_{\text {eff }}$. Problems associated with the ways of measuring $Z_{\text {eff }}$ are discussed below, along with experimental results.

Probably the most widely used method of plasma impurity identification (and hence $z_{\text {eff }}$ deternination) is spectroscopy. Many impurity lines fall in the ultraviolet, so that vacuum ultraviolet as well as visible spectra must be observed. Most ionization states of plasma impurity atons do not normally exist in nature (except perhaps in stars). Therefore, the radiation frequencies of all but the two lowest lying ionization levels are not known for most atoms. A few atoms, such as C, 0 , and Fe, have known ionization !ovels; and it is on such atoms that plasma impurity . studies are based.

Besides the problem of knowing the expected radiation frequencies, there are several difficulties in spectroscopically determining the amount of various plasma impurity species. One such difficulty is establishing the excitation rates for optical transitions. Since these rates are not generally measured, they must be inferred by theoretical calculations. A second problem is the detection of lines which are of weak intensity because of a large number of transition possibilities. A third problem is spectrometer intensity calibration in the ultraviolet. A fourth is that of obtaining and unfolaing srectral intensities as a iustiotion of radius. Taken together, these $p$ sblems result in an uncertainty factor of $\varangle 5$ in determining the amount of impurities.

In spite of these difficulties, spectroscopy provides a unique tool for the identification of impurities. Impurity species present in ORMAK [6] are given in Table I, along with their lirely origin and estimates of their abundance. 
Nother diagnostic often used to deteraine $2_{\text {eff }}$ is some form of $x$-ray detector. $X$-ray enission comes from brensstrahlung, recombination, and line radiation, i.e. free-free, free-bound, and bound-bound electron transitions. The intensity of the bremsstrahlung radiation is proportional to $\sum_{i} n_{i} n_{e} z_{i}^{2}[7]$, so the bulk of the radiation is enitted from the region of highest density. In practice wost x-rays come from a small volume, making this a "point" moasurement.

If bremsstrahlung were the only photon-producing process, measurement of the absolute intensity of $x$-rays enitted from a given volume would give $z_{\text {eff }}$ directly, provided $n_{e}$ and $T_{e}$ were known. Unforturately, recombination radiation is usually more intense than bromsstrahlung; this indicates a need to know which impurities are piesnnt and what their charge states are as a function of radius. Thus the answor one gets depends on the assumed impurities. Another problem arises when runaway ciectrons are present. Photons generated by the runaways can add a high energy tail to the $x$-ray spectrum which has nothing to do with impurities. Line radiation from bound-bound transitions represents still another couplication, and signals from such transitions must be subtracted if presient.

$x$-ray measurements of $Z_{\text {eff }}$ have been made in ORMAK using both a soft $x$-ray detector [8] and a PIN diode array. The latter detector gives spatially resolved information, an example of which is shown in fig. 1. Two cases are plotted, one assuming oxygen as the impurity and the other assuning iron as the contaminant. Despite differences having to do with the nature of the impurity, it can be seen that there is no strong central accuaulation, but rather a general increase of $z_{\text {eff }}$ with time throughout the plasma. Data taken over a wide range of plasma currents indicace, by 
comparison with resistivity measurements mentioned below, that the principal impurities present at low currents are light impurities (or a aixture of light and heavy impurities), while heavy impurities dominate at high currents.

A third common method of measuring $Z_{\text {eff }}$ is to measure the plasma resistance and to relate this directly to $z_{\text {eff }}$ by the Spitzer formula [9], assuming $T_{e}$ and $n_{e}$ are known and including both neoclassical terms to account for trapped particles not carrying current and the bootstrap current. These corrections decrease $Z_{\text {eff }}$ by 20 to $30 \%$ below the uncorrected $z_{\text {eff }}^{S p}$. While this procedure sounds straightforward, there are a number of complications. First of all, only the overall plasma resistance is measured; but the resistivity is a function of radius $R_{p}(r)=\frac{V_{p}}{2 \pi R J(r)}$. where $R_{p}(r)$ is the plasma resistivity, $V_{p}$ is the plasma voltags drop induced by the transformer, $R$ is the major radius, and $J(r)$ is the current density in the plasma. Upon locating the mode-rational surfaces $[10](q=$ $1,2)$ by means of spatially resolved $x$-ray fluctuation measurements, it is possible to infer the current density profile of the plasma. Howevar, the $Z_{\text {eff }}$ obtained depends sensitively upon the exact radial location of the $q=2$ surface, as shown in Fig. 2. The $Z_{\text {eff }}$ one gets also depends somclihat or the ispurity model, i.e. the impurity species and the ionization states as a function of radius. Another uncertainty inherent in this measurement arises from assuming that all resistance comes from collisions. Plasma turbulence also causes resistance which cannot be separated from the measurements, and so an overestimate of $Z_{\text {eff }}$ results. 
A fourth method used to determine $z_{\text {eff }}$ is to measure the chargeexchange energy spectrum of injected ions as they slow down in the plasma. Upon slowing down, their pitch angle with respect to the magnetic field changes due to scattering by impurity ions (as well as by $\mathrm{H}^{+}$ions). By the time these ions have been scattered by $90^{\circ}$ from their injected direction, their energy distribution is a function of $\mathrm{z}_{\mathrm{eff}}$, which can be calculated by means of the Fokker-Planck equation. The result can be compared with experiment, as shown in Fig. 3 for scattering from the center of the plasma. Recently, nonlocal calculations have been made which take into account scattering all along the chord viewed by the charge-exchange analyzer, and which assume a radial dependence for $Z_{\text {eff }}$.

This scattering technique seems to be straightforward, but there are difficulties both with the theory and the experimental measurements. The major theoretical unknown is that of trapped particle effects. The Fokker-Planck calculations do not, at present, take into account ion orbit trapping which leads to a radial variation in the ion scattering. This can be important, since ions scattered by $\sim 90^{\circ}$ are trapped; and it is these ions which are energy analyzed to determine $z_{\text {eff }}$ Experimentally, the number of injected particles and the background neutral density must both be known for accurate calibration. For this purpose it is necessary to measure the number of charge-exchanged beam ions as a function of energy, both parallel and perpendicular to magnetic field lines.

\section{Oxygen Discharge Pre-cleaning}

Encouraged by the results of laboratory tests [4] using glow discharges, we have tried discharge pre-cleaning in ORMAK. As with previous discharge pre-cleaning which used $\mathrm{H}_{2}$ gas, this cleaning involves $120-\mathrm{Hz}$ intermittent 
cathode-less discharges run in the same manner as regular tokamak shots, but with much reduced fields, current, and duration.

The results of switching from $\mathrm{H}_{2}$ to $\mathrm{O}_{2}$ discharge pre-cleaning [6] are shoum in Fig. A for shots of equal plasma current. Ion and electron temperatures increased, there was an increase in plasma density, and there was less $H_{B}$ and $C$ III refluxing light. The carbon concentration decreased with continued discharge pre-cleaning and was believed to be below 1\%. A comparison between the lines observed with $\mathrm{H}_{2}$ and $\mathrm{O}_{2}$ pre-cleaning is shown in Fig. 5. Note that with $\mathrm{O}_{2}$ pre-cleaning the carbon lines disappeared and many higher ionization states of oxygen were present, indicating higher electron temperatures.

It is not clear how oxygen pre-cleaning affected the amount of oxygen in the plasma. Figure 6 is a record of the oxygen lines 0 III - 0 VI as a function of time during the discharge. In each case oxygen lines were "burned" through faster and their peaks were lower with $\mathrm{O}_{2}$ pre-cleaning as opposed to $\mathrm{H}_{2}$ pre-cleaning.

Similar comparisons between $\mathrm{H}_{2}$ and $\mathrm{O}_{2}$ discharge pre-cleaning showed Au I, Fe II, Be II, and Si III impurity ionization states to be reduced throughout the discharge. Under the wall conditions tested, $\mathrm{O}_{2}$ discharge cleaning was effective in removing several impurity species, higher electron and ion temperatures were obtained, plasma currents were increased, and the operating parameter window was enlarged.

\section{Power to the Wall and $\Sigma_{\text {eff }}$ Scaling}

The integrated flux of particles and photons to the wall was monitored by a pyroelectric detector [12]. The response time of this detector was about $100 \mu \mathrm{sec}$, so that power measurements as a function of time were 
possible. Most of the power was emitted as line radiation, with a smaller but nonnegligible fraction as charge-exchanged neutrals. Power measurements as a function of time are show in Fig. 7 for three different plasma currents. During the middle portion of the discharge, when power balances were in equilibrium, 60-75\% of the power reached the walls, the remainder being deposited on the iimiter. The top $\left(I_{p}=140 \mathrm{kA}\right)$ curve in Fig. 7 shows an abrupt increase at $\sim 50 \mathrm{msec}$ due to an injection of silicon. This silicon was injected by a pulse of the Thomson scattering laser striking a glass dump; it caused a power burst as a result of increased line radiation.

- A plot of $z_{\text {eff }} \times \bar{n}_{e}$ vs the plasma current, where $Z_{\text {eff }}$ is the central value, is shown in Fig. 8. The shaded area encloses values of $z_{\text {eff }}^{\text {Sp }}$ derived directly from measurements of the plasma resistance [13]. The bottom line represents data from $x$-ray measurements. Since $z_{\text {eff }} \times \bar{n}_{e}$ scales linearly with $I_{p}, Z_{e f f} \sim I_{p} / \bar{n}_{e}$, i.e. $Z_{\text {eff }}$ increases with plasma current and decreases with plasma density, indicating a constant amount of impurities at a given power level.

\section{Summary}

Attempts have been made to correlate impurities on the surface of the ORMAK liner with plasma impurities. At present neither surface studies nor plasma impuricy studies are capable of shot-to-shot measurements, so that results to date are necessarily qualitative. The clearest correlations are those between $\mathrm{H}_{2}$ and $\mathrm{O}_{2}$ discharge pre-cleaning, where both wall contaminants (as tested in the laboratory) and plasma contamination spectra were observed to change. If more detailed comparisons are to be made, much work must be done to develop both surface physics and plasma impurity diagnostics. 


\section{Acknowledgments}

We would like to thank W. R. Wing, J. A. Rome, and R. C. Isier for valuable discussions; J. L. Dunlap for data on location of the mode-rational surfaces; and R. E. Clausing and L. C. Emerson for AES anaiysis of various materials removed from ORMAK. P. H. Edmonds and H. E. Ketterer were responsible for machine operation. We gratefully acknowledge the continuing support of L. A. Berry and J. F. Clarke in carrying out these experiments.

\section{References}

[1] J. E. Simpkins, Proc. 5th Symposium on Engineering Problems of Fusion Research, Princeton University (1973) p. 385 .

[2] R. J. Colchin et al., J. Nucl. Mater. 53 (1974) 25.

[3] R. E. Clausing et al., paper in these proceedings.

[4] R. E. Clausing et al., J. Vac. Sci. Technol. 13, No. 1 (1976).

[5] L. A. Berry et al., Plasma Physics and Controlled Nuclear Fusion Research, Vo1. I (IAEA, Vienna, 1975) p. 101.

[6] R. V. Neidigh and L. J. Nugent, Bull. Am. Phys. Soc. 20 (1975) 1300.

[7] T. F. Stratton in Plasma Diagnostic Techniques (Academic Press, Inc., New York, 1965) ed. by R. H. Huddleston and S. L. Leonard, p. 359.

[8] W. R. Wing, Bul1. Am. Phys. Soc. 18 (1973) 1254.

[9] L. Spitzer, Jr., Physics of Fully Ionized Gases, 2nd ^d., (John Wiley, N. Y., 1962).

[10] L. A. Artsimovich, Nuc1. Fusion 12 (1972) 215.

[11] L. A. Berry et al., Plasma Physics and Controlled Nuclear Fusion Research, Vol. 1 (IAEA, Vienna, 1974) p. 113.

[12] C. E. Bush et al., Bull. Am. Phys. Soc. 20 (1975) 1299.

[13] M. Murakami et al., Bul1. Am. Phys. Scc. 20 (1975) 1299. 
Table I. ORMAR Impurities

\begin{tabular}{|c|c|c|}
\hline Element & Origin & $\begin{array}{c}\text { Element } \\
\text { Abundance }\left(\% \mathbf{n}_{\mathbf{e}}\right)\end{array}$ \\
\hline He & diagnostics & trace \\
\hline $\mathrm{Be}$ & $x$-ray window & $<1.0$ \\
\hline c & pump oil & $>5.0 \rightarrow<1.0$ \\
\hline $\mathbf{N}$ & air & trace \\
\hline c & discharge cleaning & $1-2$ \\
\hline $\mathrm{N} 3, \mathrm{Cl}, \mathrm{Ca}$ & perspiration & $<0.1$ \\
\hline Si & quartz windows & $<1.0$ \\
\hline $\mathbf{s}$ & abundant element & $\sim 0.1$ \\
\hline $\mathrm{Cr}, \mathrm{Mn}, \mathrm{Ni}$ & 304 SS & \\
\hline $\mathrm{Fe}$ & 304 SS & $<1.0$ \\
\hline $\mathrm{Cu}$ & injectors & \\
\hline $\mathbf{A g}$ & injectors & \\
\hline $\mathbf{W}$ & limiter & \\
\hline $\mathbf{P t}$ & liner plate & \\
\hline $\mathbf{A u}$ & liner plate & \\
\hline
\end{tabular}




\section{FIGURE CAPTIONS}

Fig. 1. Time evolution of $Z_{\text {eff }}$ profiles measured by a PIN diode array for $I_{p}=175 \mathrm{kA}$ discharges. Oxygen was assumed to be the impurity in the leit-hand plot and iron was assumed on the right. Upward-pointing arrows indicate maximum possible $z_{\text {eff }}$.

Fig. 2. Values of $z_{\text {eff }}$ as a function of radius for four positions of the $q=2$ mode-rational surface. Although $z_{\text {eff }}$ is sensitive to the position of the $q=2$ surface, it is rolatively insensitive to the $q=1$ location.

Fig. 3. Parallel and perpendicular energy distributions of captured beam ions as they slow down in energy and scatter in pitch angle. The best data fit gives $Z_{\text {eff }} \simeq 4$.

Fig. 4. Comparison of oxygen and hydrogen discharge pre-cleaning on plasma characteristics. Plots on the left show increases in the plasma temperature and density after oxygen pre-cleaning, and plots on the right show decreases in $\mathrm{H}_{\beta}$ and $\mathrm{C}$ III light.

Fig. 5. Spectrum of strong lines in the ultraviolet (10-300 A) after hydrogen and oxygen discharge pre-cleaning. Fc XV and Fe XXII lines appear only after $\mathrm{O}_{2}$ discharge cleaning. The Fe XXII 1 ine appears as a faint smudge. Hundreds of weaker lines are not reproduced.

Fig. 6. Spectral intensity (normalized by the electrm: density) of several oxygen lines during the first $20 \mathrm{msec}$ of hydrogen and oxygen precleaned discharges.

Fig. 7. Power to the wall as a function of time for plasma currents of 140,122 , and $100 \mathrm{kA}$. The corresponding power inputs to the plasma were 411,333 , and $234 \mathrm{kw}$. 


\section{Figure Captions (cont.)}

Fig. 8. $z_{\text {eff }} \times \bar{n}_{e}$ as a function of the plasma current. The shaded area represents the range of $z_{\mathrm{eff}}^{\mathrm{Sp}}$ as derived from the Spitzer resistivity formula with no allowance for trapped particles or bootstrap currents. The dashed line is $z$ fit to $z_{\text {eff }}^{X}$ determined from $x$-ray measurements. Open circles are for oxygen, solid circles are for iron, and triangular points indicate that an impurity sacurated plasma is required for the assumed impurity species. 


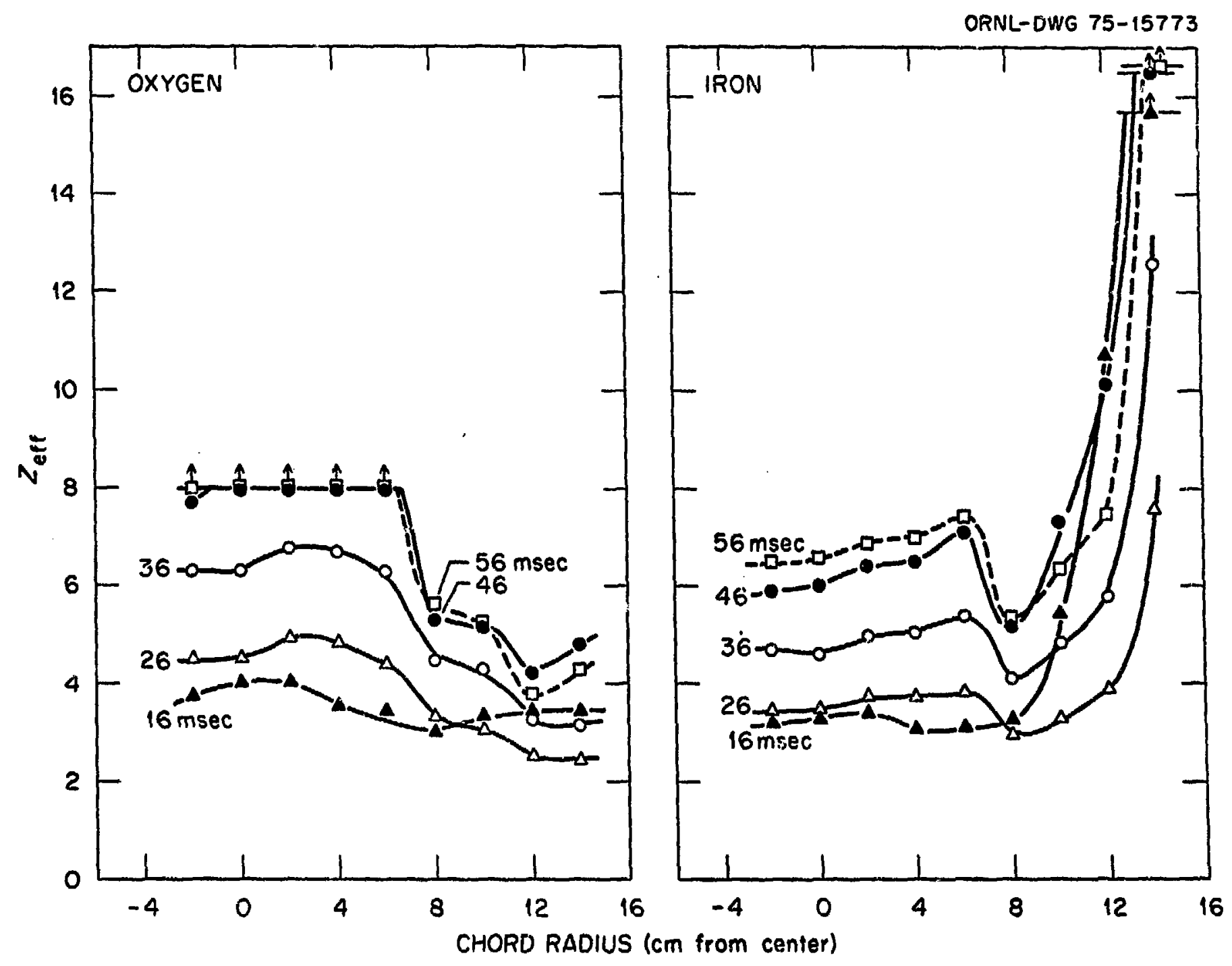




\section{IRON IMPURITY MODEL}

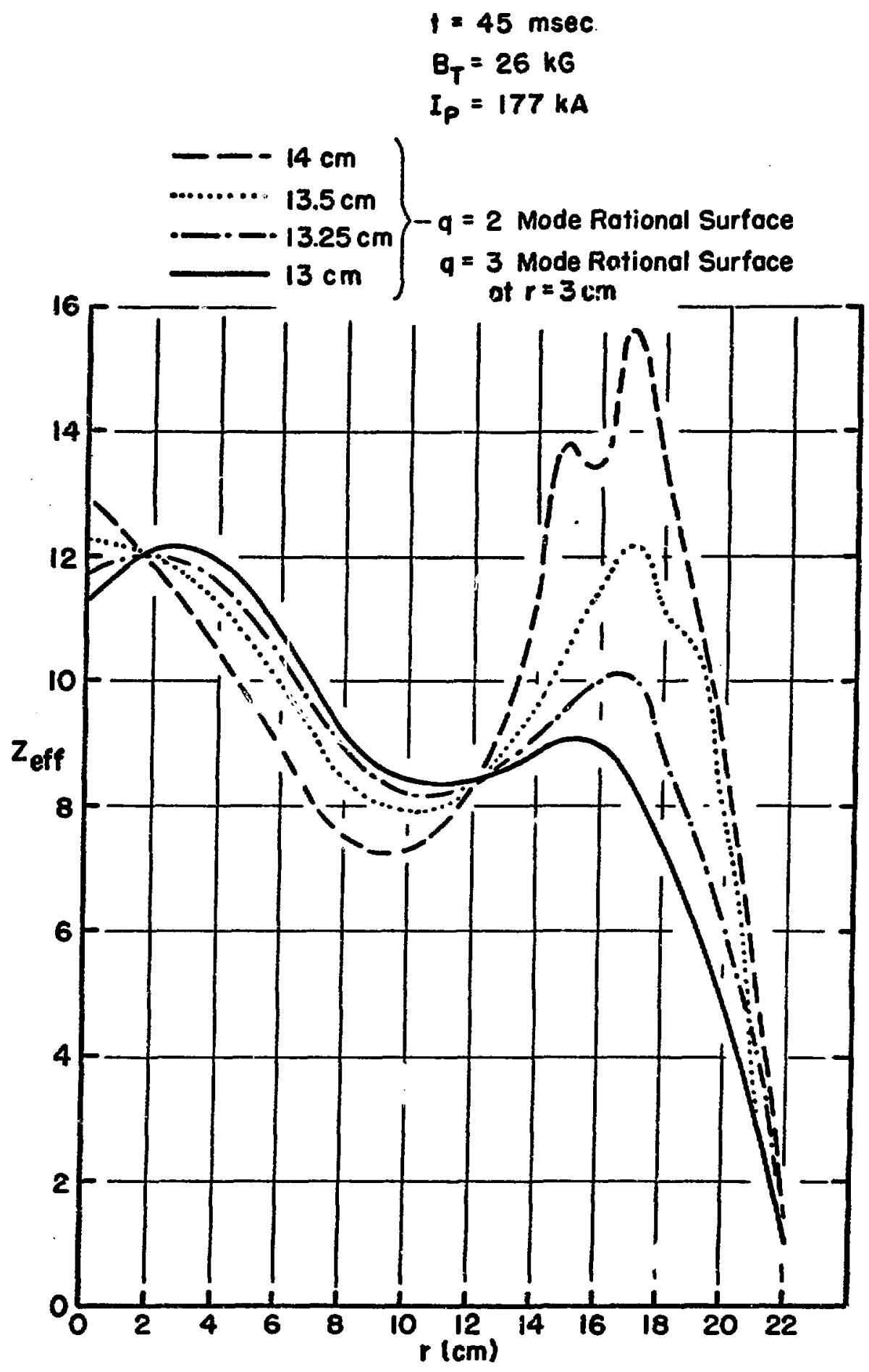



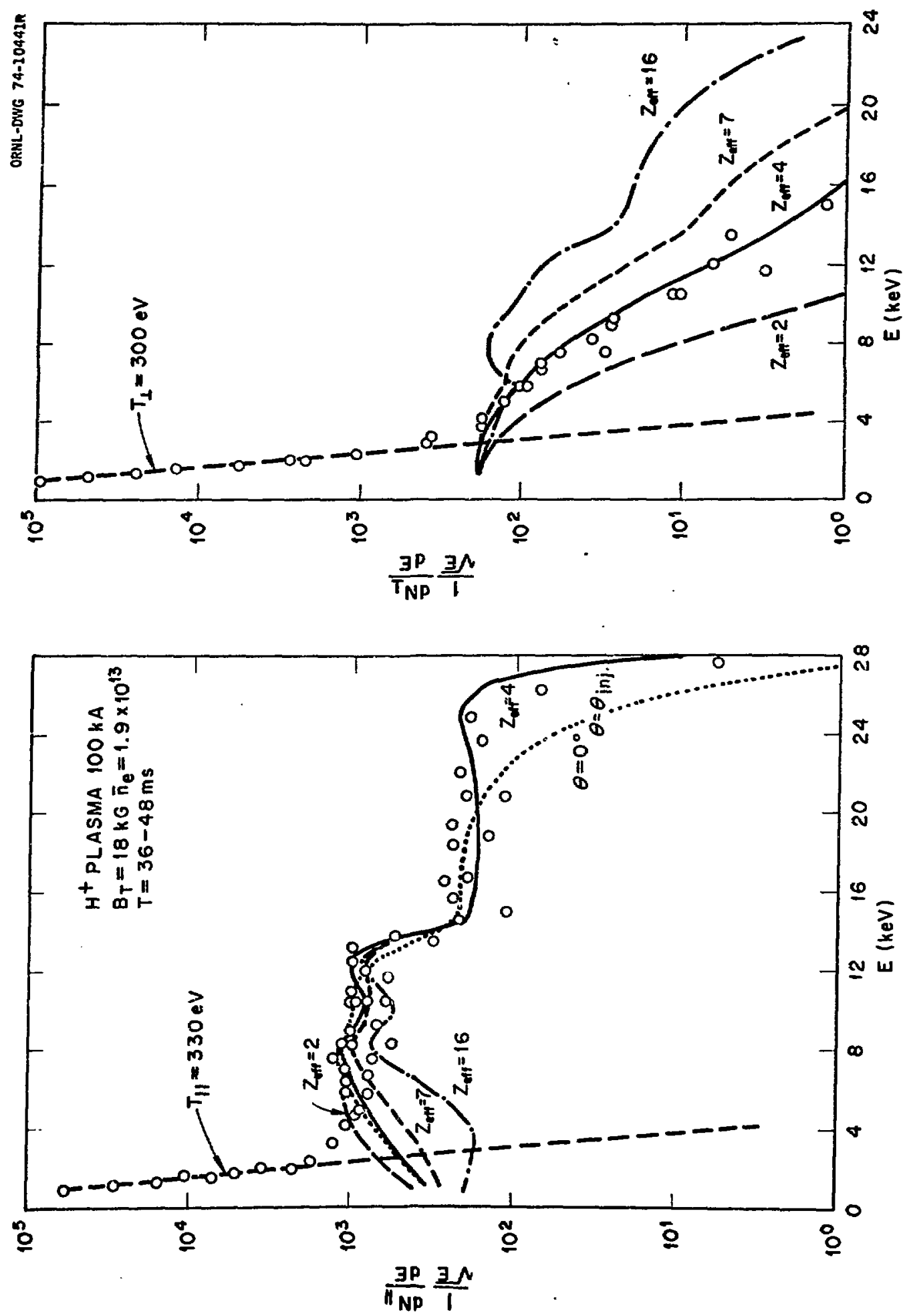


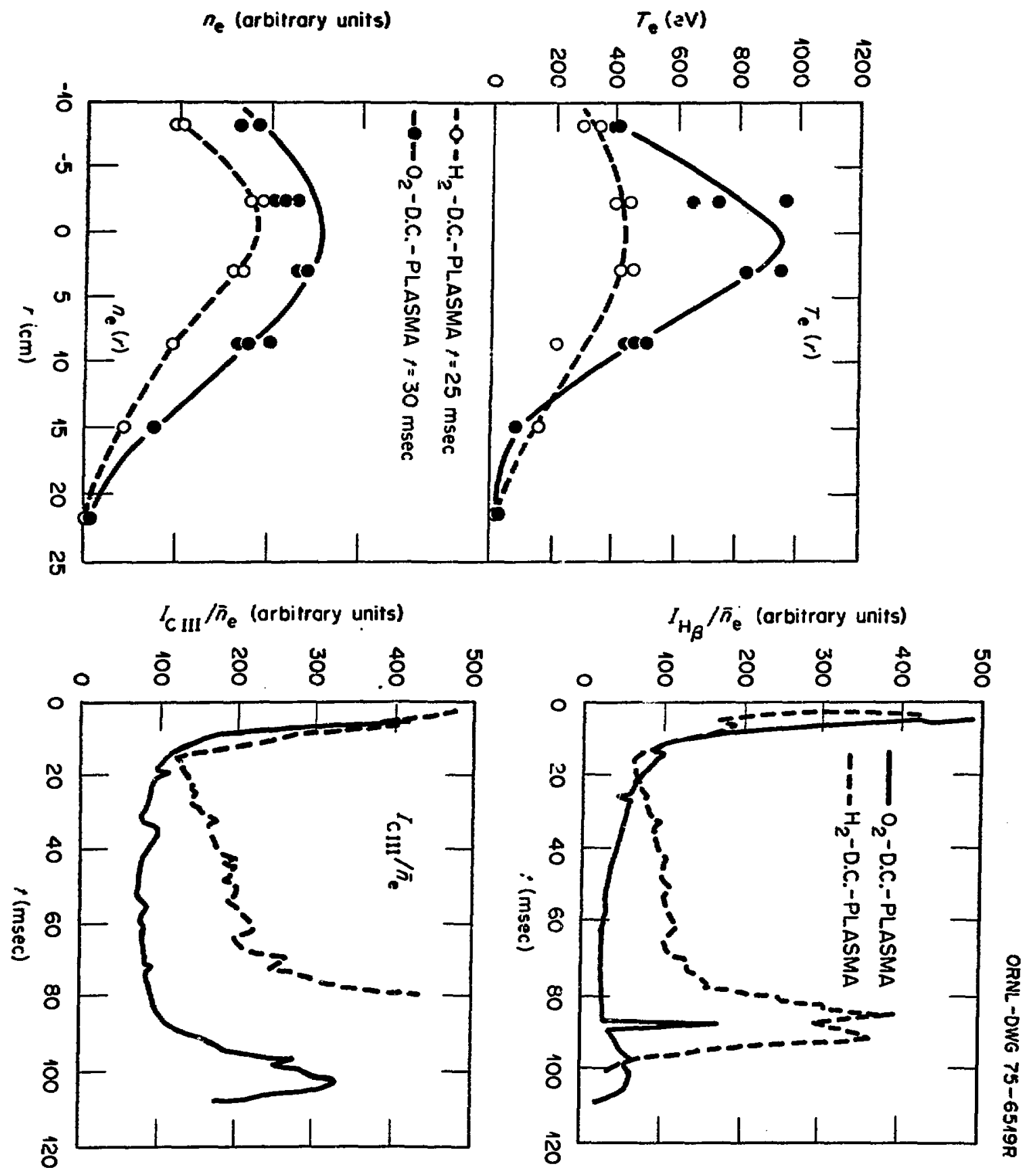


ORNL-D:JF 76-3105

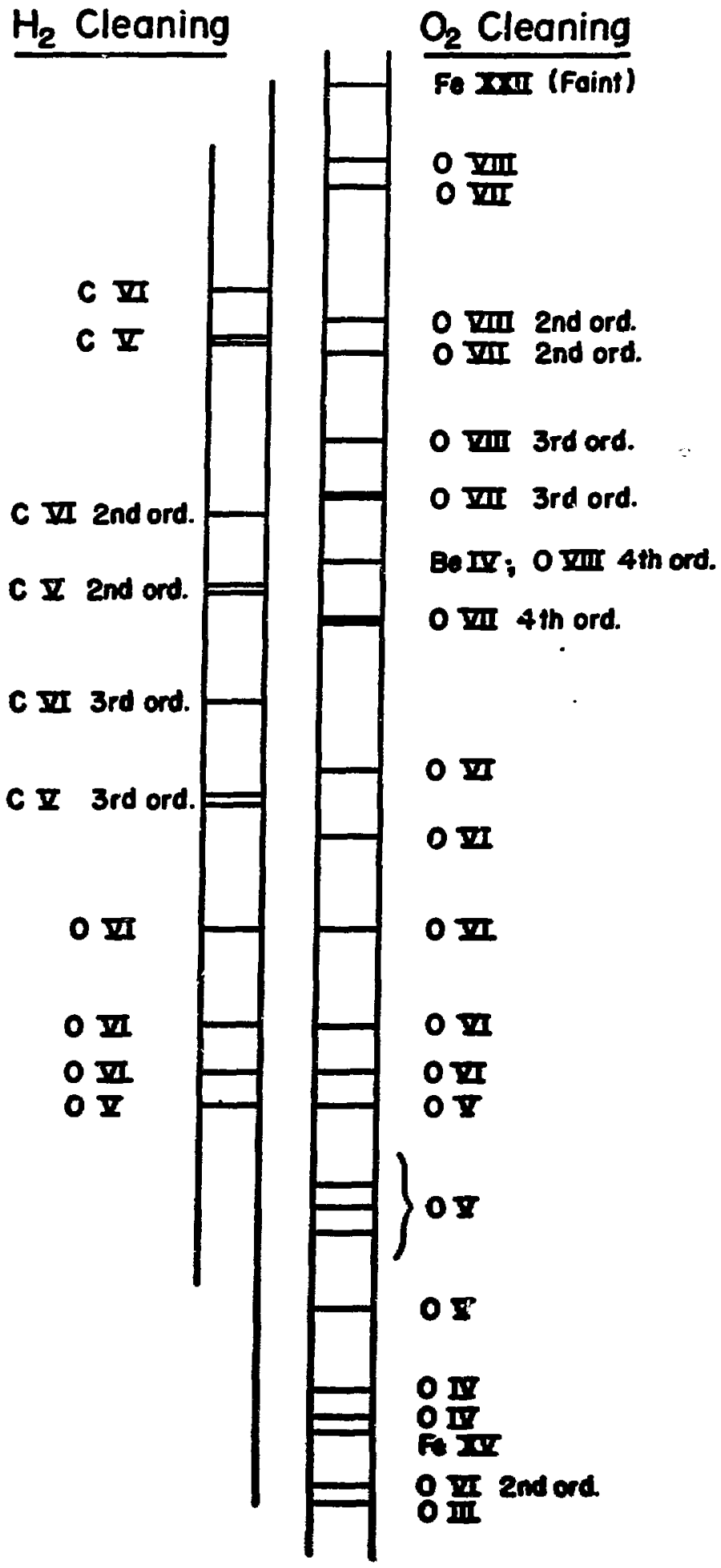




$$
\text { ORNL-DWG 75-13176R }
$$

-- DISCHARGE CLEANINS IN $\mathrm{H}_{2}$
- DISCHARGE CLEANING IN $\mathrm{O}_{2}$

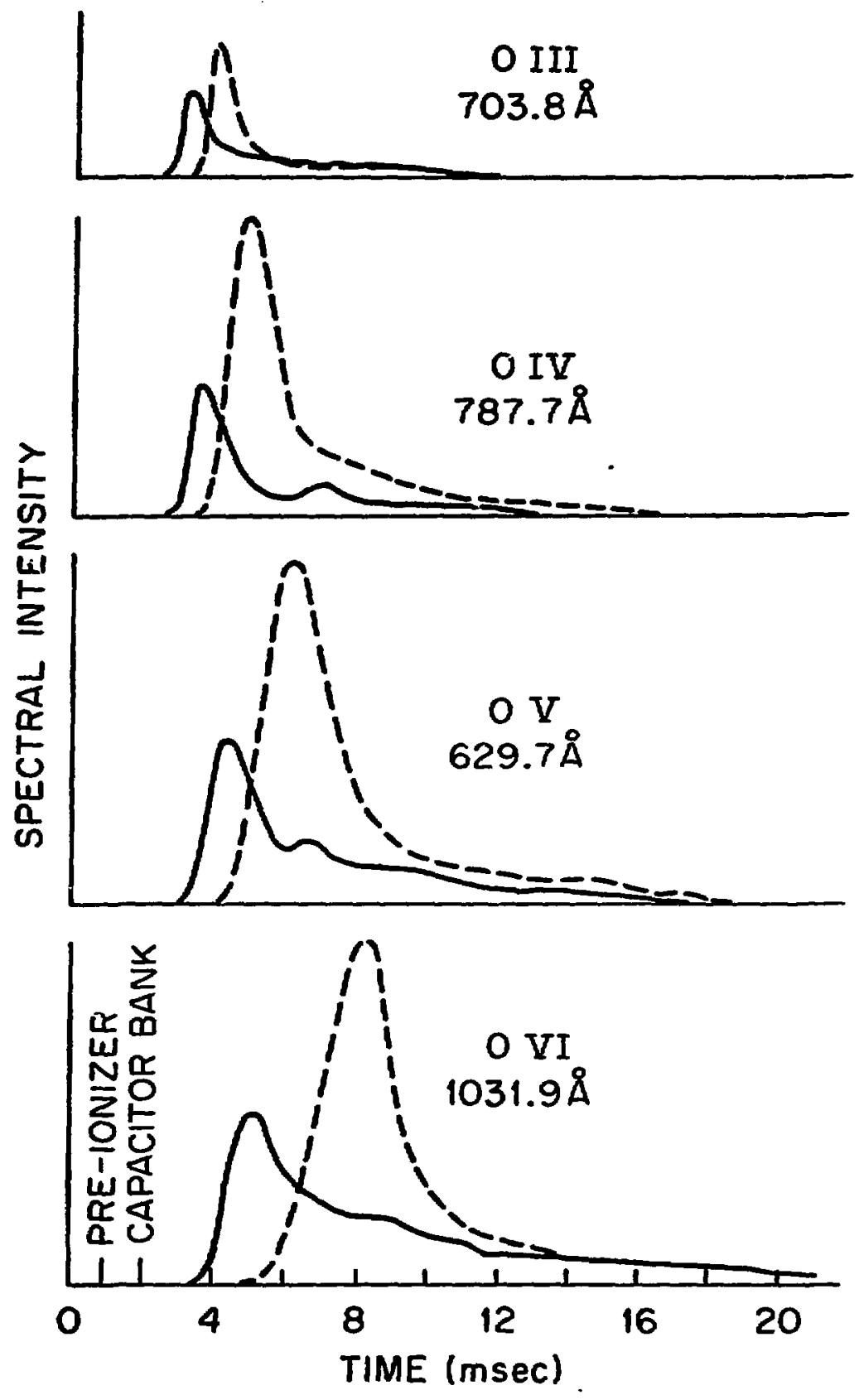




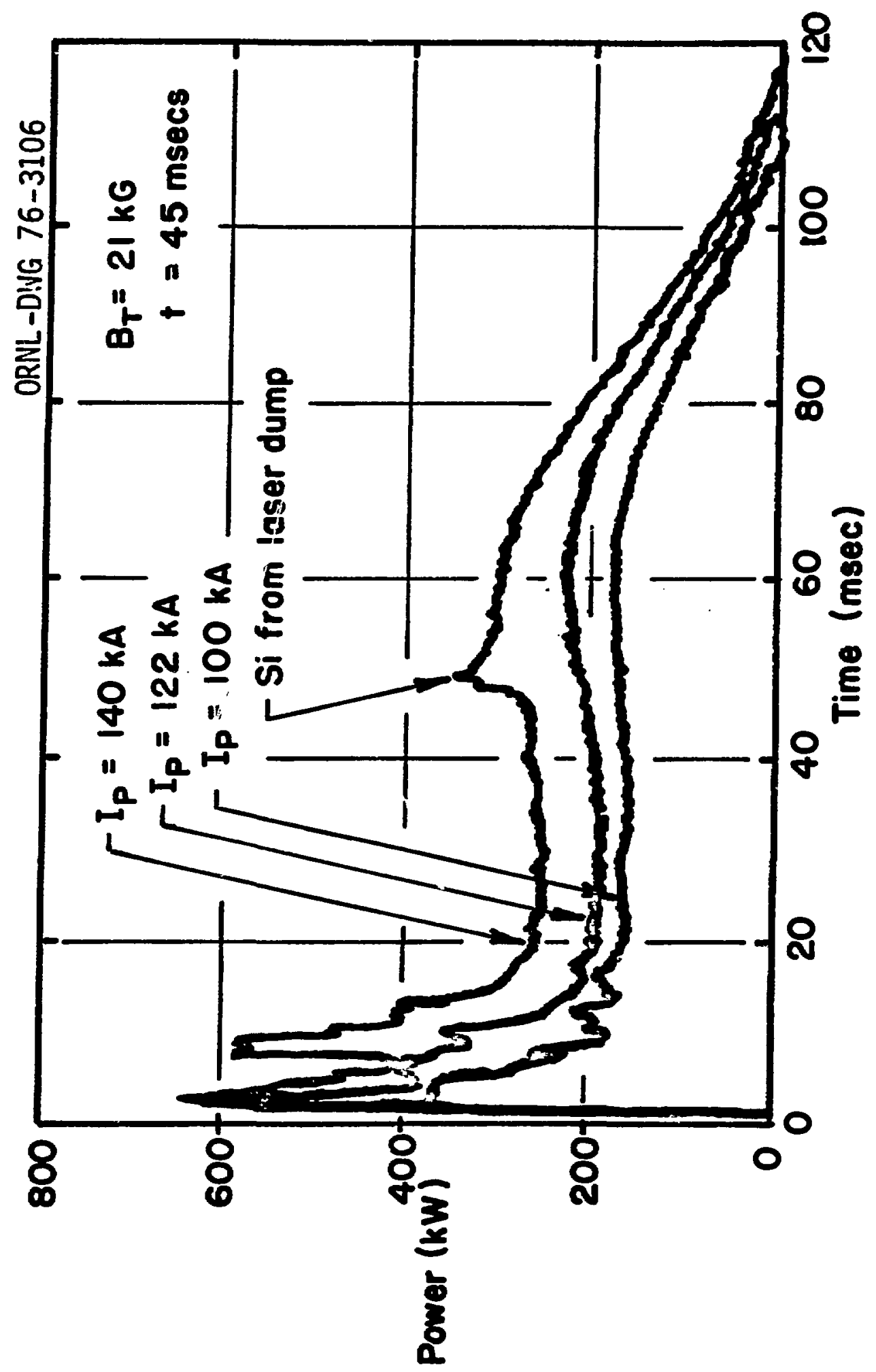

